\title{
Active Surveillance Perspectives of Radiation Oncologists, Medical Oncologists and Urologists in the Treatment of Prostate Cancer
}

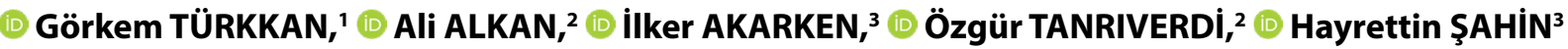

'Department of Radiation Oncology, Muğla Sıtkı Koçman University Faculty of Medicine, Muğla-Turkey

2Department of Medical Oncology, Muğla Sıtkı Koçman University Faculty of Medicine, Muğla-Turkey

${ }^{3}$ Department of Urology, Muğla Sıtkı Koçman University Faculty of Medicine, Muğla-Turkey

\begin{abstract}
OBJECTIVE
To evaluate the perspectives of radiation oncologists (ROs), medical oncologists (MOs) and urologists (UROs) towards active surveillance (AS) in the management of prostate cancer (PCa).

\section{METHODS}

A questionnaire with total of 24 questions was sent out via e-mail to the physicians. 244 participants completed the questionnaire. Pearson Chi square test and multivariable logistic regression models were used to identify physicians' characteristics and attitudes about AS.

RESULTS

There were 129 UROs (52.9\%), 76 ROs (31.1\%) and 39 MOs (16\%) in the study population. The analysis of the important factors while considering AS showed that prostate cancer risk group (85.7\%) was the most commonly considered criteria, followed by patient's request and compliance $(84.8 \%)$, life expectancy (76.2\%) and sexual activity of the patient (34.8\%). The AS was recommended by $86.8 \%$ of UROs, $77.6 \%$ of ROs and $61.6 \%$ of MOs ( $\mathrm{p}=0.002)$. In multivariate analysis, practicing as ROs $(\mathrm{p}=0.031)$ or UROs $(\mathrm{p}<0.001)$, working in a reference hospital $(\mathrm{p}=0.006)$ and having an uro-oncology board $(\mathrm{p}=0.031)$ were found to be associated with more recommendations for AS.
\end{abstract}

\section{CONCLUSION}

More clinical experience and multi-disciplinary approach were associated with tendency of recommending AS. Educational sessions and uro-oncology board discussions may provide more integration of AS to our clinical practice routines.

Keywords: Active surveillance; prostate cancer; radical prostatectomy; radiotherapy; treatment strategy. Copyright $\odot$ 2021, Turkish Society for Radiation Oncology

\section{Introduction}

Prostate cancer $(\mathrm{PCa})$ is the most common malignancy in men and the $2^{\text {nd }}$ most common cause of cancer-related deaths.[1] As a result of increased prostate-specific-antigen (PSA) screening, early stage PCa cases are increasing. With more experience with low risk PCa, a conservative approach has emerged because of the worries about overdiagnosis, overtreatment and treatment-related toxicities.[2] Active surveillance (AS)/ watchful waiting (WW) have become to be used more frequently in the management of PCa. In a recent study including data of 50302 low-risk PCa patients from Surveillance, Epidemiology, and End Results database,

\section{Dr. Görkem TÜRKKAN}

Muğla Sıtkı Koçman Üniversitesi Tıp Fakültesi,

Radyasyon Onkolojisi Anabilim Dalı,

Muğla-Turkey

E-mail: gorkemturkkan@gmail.com,gorkemturkkan@mu.edu.tr 
it was reported that, from 2010 to 2015 , AS/WW rates have increased from 11.2 to $37.3 \%, 14.1$ to $45.8 \%$ and 17.6 to $46.4 \%$ in the low, middle and high socioeconomic status groups, respectively.[3]

Although there are some controversies and differences about the implementation of AS between institutions, it's mainly recommended for selected very low/ low risk and low-volume favorable intermediate risk (Gleason 3+4) PCa patients. In the literature, AS has been confirmed by various studies as a safe, appealing and effective treatment strategy.[4,5] Long-term outcomes of the prostate cancer intervention versus observation (PIVOT) trial showed no survival advantage with radical prostatectomy (RP) over observation in low risk PCa patients. [6] Consistent with PIVOT trial, the prostate testing for cancer and treatment (ProtecT) trial showed no survival benefit of RP or radiotherapy (RT) as compared to active monitoring, for patients with localized disease.[7] In contrast to above-mentioned 2 major studies, only Scandinavian prostate cancer group-4 (SPCG-4) trial showed survival benefit with RP over WW.[8] The benefit was largest in patients $<65$ years of age and in those with intermediaterisk PCa. But, it should be kept in mind that this study was performed in the pre-PSA era. Small differences in inclusion and follow-up criteria of studies may also explain this discrepancy.

Considering all these data, it is reasonable to use AS in selected cases to avoid/delay treatments and their side effects. AS has been reported to be able to reduce overtreatment and treatment costs in group of patients with low-risk PCa.[9] Therefore, awareness and attitudes of PCa specialists towards AS are quite important for the adoption of AS in the management of PCa. The literature includes different results in a limited number of studies evaluating physicians' attitudes towards AS. In a national survey in which respondents were radiation oncologists (ROs) and urologists (UROs), physicians' perceptions of possible barriers towards AS for low-risk PCa were analyzed. Prejudice of low interest of patients in AS, worries about repeated prostate biopsy necessity, biased treatment suggestions of physicians in favor of their own expertise were reported as key barriers to AS.[10] Another recent survey study was conducted with 52 respondents who are ROs, medical oncologists (MOs) or UROs. Fear of patient non-compliance and lack of awareness were the main impediments for the implementation of AS.[11]

In present study, we performed a questionnaire in Turkey to evaluate the perspectives of ROs, MOs and UROs towards AS. To the best of our knowledge, this is the largest study evaluating the attitudes of ROs, MOs and UROs towards AS in the management of PCa.

\section{Materials and Methods}

The study was conducted as a cross-sectional questionnaire study, in order to assess the attitudes of ROs, MOs and UROs towards AS in the management of PCa. Institutional Ethics Committee approved the study protocol. All procedures performed in studies involving human participants were in accordance with the ethical standards of the institutional and/or national research committee and with the 1964 Helsinki declaration and its later amendments or comparable ethical standards. Informed consent was obtained from all individual participants included in the study.

A structured questionnaire with a total of 24 questions was designed electronically. It contained dichotomous and multiple-choice questions evaluating the physicians' sociodemographic characteristics (questions 1-7), their current primary treatment preferences for low-risk PCa (questions 14-17) and their attitudes towards AS (the remaining questions). The whole questionnaire was shown in Annex File 1. Between September-October 2019, 598 physicians were invited to study by e-mails and the responses were collected. The invitation e-mails were sent 3 times in the 2 months of data collection period to enable more feedbacks.

Descriptive analyses were done using frequencies for the sociodemographic variables. In order to assess for differences in physician characteristics and questionnaire answers, bivariate analyses were conducted using Pearson chi-square test. To analyze the factors associated with recommending AS, the academic ranking was grouped as academic staff (professor, assoc./asst. professor) and others. The primary place of work was grouped into reference hospital (university/education and research hospital) and others. The parameters associated with more recommendations of AS (with $p$ value $<0.2$ ) were used for multivariate analysis. Multivariable logistic regression models were carried out to identify relevant factors of participants, which were associated with different attitudes of physicians about AS.

The Statistical Package for the Social Sciences (SPSS) version 21.0 (SPSS Inc., Chicago, Illinois, USA) was used for statistical analysis and a p-value of less than 0.05 was considered statistically significant. 


\section{Results}

A total of 244 participants (response rate: $40.8 \%$ ) completed the questionnaire. The baseline characteristics of the participants are summarized in Table 1. Most of the participants were male $(182,74.6 \%)$ and nearly half of them were between 30 and50 years of age (129, $52.9 \%)$. There were 129 UROs (52.9\%), 76 ROs (31.1\%) and 39 MOs (16\%) in the study population. The most common primary place of work was a university/education and research hospital $(160,65.6 \%)$. While half of them were specialists $(122,50.0 \%), 52.9 \%$ of them had been caring cancer patients for more than 10 years. One-fourth of the physicians (25.6\%) stated that they had never had a multidisciplinary uro-oncology board during their medical career. The participants declared that the probability of overtreatment $(61.1 \%)$ was the most challenging problem in the management of PCa. Among the participants, the asst.professor/professors ( $72.0 \%$ vs $54.3 \%, \mathrm{p}=0.04)$ and UROs $(66.7 \%$ vs $54.8 \%$, $\mathrm{p}=0.03)$ had more concerns about overtreatment. In addition, $44.7 \%, 27.9 \%$ and $20.1 \%$ of them thought that there were still problems in treatment, screening and diagnosis of $\mathrm{PCa}$, respectively. When compared to UROs $(41,31.8 \%)$, more MOs $(27,69.2 \%)$ and ROs

Table 1 The baseline characteristics of the participants

\begin{tabular}{lc} 
Characteristics & $\mathbf{n}(\%)$ \\
\hline Age (years) & \\
$\quad<30$ & $14(5.7)$ \\
$30-50$ & $171(70.1)$ \\
$\quad>50$ & $59(24.2)$ \\
Gender-male & $182(74.6)$ \\
Specialties & \\
$\quad$ Urology & $129(52.9)$ \\
$\quad$ Radiation oncology & $76(31.1)$ \\
$\quad$ Medical oncology & $39(16.0)$ \\
Academic Rank & \\
$\quad$ Professor/Assoc.Prof./Asst.Prof. & $93(38.1)$ \\
$\quad$ Specialist & $122(50.0)$ \\
Resident/Fellow & $29(11.9)$ \\
Primary place of work & \\
$\quad$ University/Education and research hospital & $160(65.6)$ \\
Public hospital & $37(15.2)$ \\
Private practice & $47(19.3)$ \\
Experience in specialty & \\
$<5$ years & $53(21.7)$ \\
5-10 years & $62(25.4)$ \\
$>10$ years & $129(52.9)$
\end{tabular}

Assoc.Prof.: Associated professor; Asst.Prof.: Assistant professor.
$(41,53.9 \%)$ thought that there were problems in terms of treatment modalities ( $\mathrm{p}<0.001)$. Additionally, 35.9\% of the MOs worried about screening in PCa $(26.3 \%$ of ROs and $26.4 \%$ of UROs, $\mathrm{p}<0.001$ ).

Almost every four out of 5 physicians (79.9\%) were suggesting AS to PCa patients in their daily practice, while $76.6 \%$ of them thought that they had enough knowledge about inclusion criterias of AS for PCa. A great majority (91\%) of the physicians declared that AS could be beneficial for selected patients. However, 47 physicians (19.3\%) worried about monitoring patients with AS and 22 physicians $(9.0 \%)$ thought that they had inadequate experience to monitor a patient with AS. The analysis of the important factors while considering AS showed that prostate cancer risk group $(85.7 \%)$ was the most commonly considered criteria, followed by patient's request/compliance $(84.8 \%)$, life expectancy (76.2\%) and sexual activity status of the patient $(34.8 \%)$. The factors considered by different specialties were summarized in Figure 1.

The AS was recommended by $86.8 \%$ of UROs, $77.6 \%$ of ROs and $61.55 \%$ of MOs ( $\mathrm{p}=0.002)$ (Table 2). More UROs thought that they had enough knowledge about AS ( $82.9 \%$ vs $69.6, \mathrm{p}=0.01)$. The ones working in university/education and research hospital had more tendency to recommend AS ( $85.0 \%$ vs $70.2 \%, \mathrm{p}=0.006$ ). In addition, academic staff recommended AS more often when compared to others ( $88.2 \%$ vs $74.8, \mathrm{p}=0.008$ ). The physicians who had an uro-oncology board experience in their career $(86.7 \%)$ recommended AS more when compared to those who didn't $(72.4 \%)$ have an uro-oncology board experience $(\mathrm{p}=0.004)$. In multivariate analysis, practicing as ROs (OR: 2.7 , CI95\% 1.09-6.7, $\mathrm{p}=0.031$ ) or UROs (OR: 0.03, CI 95\% 3.3-

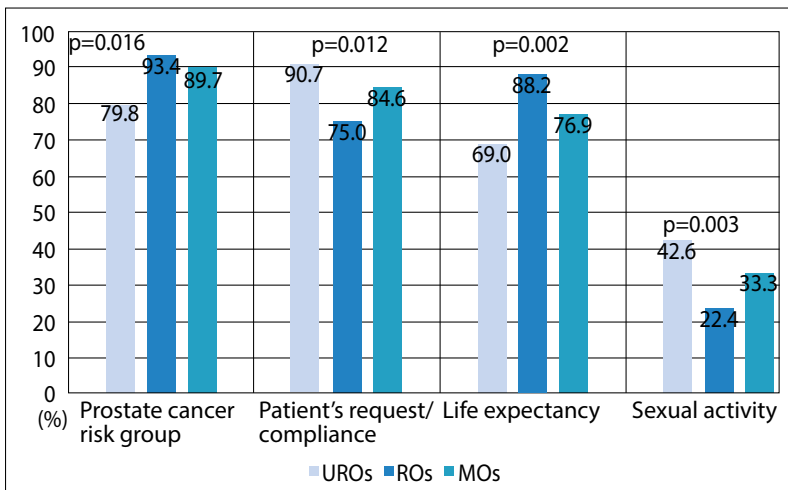

Fig. 1. The results of the factors while considering active surveillance in terms of different specialties. UROs: Urologists, ROs: Radiation oncologists, MOs: Medical oncologists. 
Table 2 The factors effecting recommendations for active surveillance

\begin{tabular}{|c|c|c|}
\hline Parameters & $\begin{array}{c}\text { Recommending AS, } \\
\text { n (\%) }\end{array}$ & $\mathbf{p}$ \\
\hline \multicolumn{3}{|l|}{ Age (years) } \\
\hline$<30$ & $12(85.7)$ & 0.52 \\
\hline $30-50$ & $133(77.8)$ & \\
\hline$>50$ & $50(84.7)$ & \\
\hline \multicolumn{3}{|l|}{ Gender } \\
\hline Male & $147(80.8)$ & 0.34 \\
\hline Female & $48(77.4)$ & \\
\hline \multicolumn{3}{|l|}{ Specialties } \\
\hline Urology & $112(86.8)$ & 0.002 \\
\hline Radiation Oncology & $59(77.6)$ & \\
\hline Medical Oncology & $24(61.5)$ & \\
\hline \multicolumn{3}{|l|}{ Academic Rank } \\
\hline Professor/Assoc.Prof./Asst.Prof. & $83(88.2)$ & 0.034 \\
\hline Specialist & $91(74.6)$ & \\
\hline Resident/Fellow & $22(75.9)$ & \\
\hline Academic staff & $82(88.2)$ & 0.008 \\
\hline Others & $113(74.8)$ & \\
\hline \multicolumn{3}{|l|}{ Primary place of work } \\
\hline $\begin{array}{l}\text { University/Education and } \\
\text { research hospital }\end{array}$ & $136(85.0)$ & 0.02 \\
\hline Public hospital (Non-teaching) & $27(73.0)$ & \\
\hline Private practice & $32(68.1)$ & \\
\hline Reference hospital & $136(85.0)$ & 0.006 \\
\hline Other & $59(70.2)$ & \\
\hline \multicolumn{3}{|l|}{ Experience in oncology field } \\
\hline Less than 5 years & $41(77.4)$ & 0.44 \\
\hline $5-10$ years & $47(75.8)$ & \\
\hline More than 10 years & $107(82.9)$ & \\
\hline \multicolumn{3}{|l|}{ Uro-oncology board } \\
\hline Present & $111(86.7)$ & 0.004 \\
\hline Absent & $85(72.4)$ & \\
\hline
\end{tabular}

AS: Active surveillance; Assoc.Prof.: Associated professor; Asst.Prof.: Assistant professor.
24.6, $\mathrm{p}<0.001$ ), working in a reference hospital (OR: 3.03, CI 95\% 1.3-6.7, $\mathrm{p}=0.006$ ) and having an uro-oncology board experience (OR: 2.2, CI 95\% 1.07-4.8, p= 0.031 ) were associated with more recommendations for AS (Table 3).

Majority of the physicians (88.9\%) had concerns about AS. The most common concern was the noncompliance of patients (79.1\%), followed by the risk of losing a curative treatment opportunity $(34.8 \%)$. Additionally, $25.8 \%$ and $18.9 \%$ of the physicians had concerns of local progression risk and lymphatic/systemic metastasis risk, respectively. A minority of participants $(19,7.8 \%)$ had concerns about inadequacy of data in literature. The concerns of different specialties were summarized in Figure 2.

As a primary treatment of PCa patients with life expectancy of $\geq 10$ years, $54.1 \%$ and $2 \%$ of the physicians recommended AS for very low/low risk and favorable intermediate risk groups, respectively. For favorable intermediate risk patients, RP (60.2\%) was the most commonly recommended primary treatment option, followed by RT (36.9\%). On the other hand, as a primary treatment of PCa patients with life expectancy of $<10$ years, $52.5 \%$ and $10.2 \%$ of the physicians recommended AS for very low/low risk and favorable intermediate risk groups, respectively. The most commonly recommended primary treatment option was RT (70.9\%) for favorable-intermediate risk patients with life expectancy of $<10$ years.

For localized disease, $75 \%$ of the physicians preferred to use multiparametric prostate magnetic resonance imaging (MPMRI) to make a decision for AS. A great majority of the physicians (95.1\%) used PSA test for AS protocol. Others were MPMRI (69.7\%), prostate biopsy (65.6\%) and digital rectal examination (65.2\%), respectively.

Table 3 Multivariate analysis of factors associated with recommendation for active surveillance

\section{Variable}

\section{Recommending Active Surveillance}

\begin{tabular}{lccc} 
& OR & Cl (95\%) & p \\
\cline { 2 - 4 } & & & \\
Specialties & & & 0.031 \\
$\quad$ Medical Oncology* & 2.7 & $3.09-6.7$ & $<0.001$ \\
$\quad$ Radiation Oncology & 9.03 & $0.8-4.6$ & 0.13 \\
$\quad$ Urology & 1.8 & $1.3-6.7$ & 0.006 \\
Being an academic staff & 3.03 & $1.07-4.8$ & 0.031 \\
Working in a reference hospital & 2.2 & & \\
Having an uro-oncology board & & & \\
\hline
\end{tabular}

*reference parameter; OR: Odds ratio; Cl: Confidence interval. 
Unless clinically indicated, most of the physicians (69.7\%) suggested assessing AS patients in every 3 months and similarly, $66.8 \%$ of them suggested an evaluation with PSA test in every 3 months. There was no consensus for the evaluation interval with digital rectal examination (DRE). While $38.1 \%$ of the physicians suggested an evaluation with DRE in every 3 months, $35.7 \%$ of them suggested DRE in every 6 months. 126 physicians (51.6\%) suggested prostate biopsy annually, and $50.8 \%$ of the physicians used MPMRI annually.

\section{Discussion}

Active surveillance has become an increasingly used treatment strategy for low risk PCa. Our study showed that a great majority of PCa physicians (ROs, MOs and UROs) in Turkey think that AS could be beneficial for selected PCa patients and, AS was being suggested by every 4 out of 5 PCa physicians.

A recent study with a small number of participants (52 physicians in total, including 5 ROs, 8 MOs and 39 UROs) reported that AS was more commonly suggested by UROs, physicians with $>15$ years in practice and physicians working in university hospitals. [11] Consistent with this study, our study showed that physicians working in a reference hospital were more likely to suggest AS. Additionally, UROs seemed to be a pillar support for the implementation of AS in PCa treatment and ROs were recommending AS more than MOs. Unlike this study, longer-term practice in oncology was not related to more recommendation for AS. Moreover, being a member of the academic staff and having an uro-oncology board in medical institution were associated with more AS suggestion of physicians. Briefly, our results demonstrated that being UROs or ROs rather than MOs and working in more academic or multidisciplinary conditions may lead PCa physicians to offer AS more.

In another study, age and comorbidities were seen as the only patient characteristics which might influence all physicians on their treatment recommendation of AS. Patient's willingness and ability to follow an AS protocol, patient's treatment preferences and life expectancy were major factors influencing physicians' treatment recommendation of AS.[12] In our study, prostate cancer risk group and patient's request/compliance were the main considerations of physicians for the implementation of AS.

Although physicians' AS suggestion rates were up to $80 \%$ in our study, $88.9 \%$ of the physicians had at least one concern about AS, most commonly "non-compli-

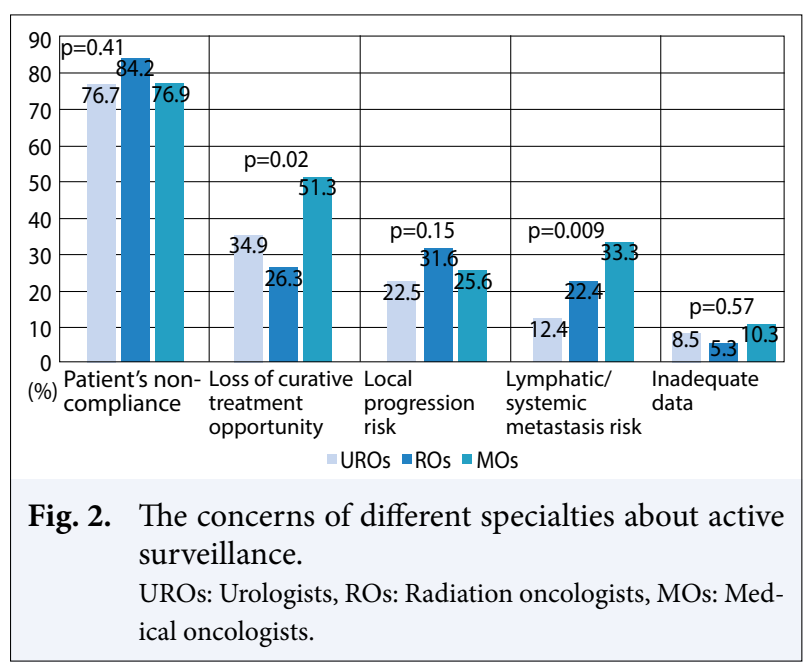

ance of patients". Patient non-compliance may be associated with many factors. In their national study, Kim et al.[10] reported a substantive rate of ROs and UROs perceive that several newly diagnosed low-risk PCa patients desire some form of primary treatment and not interested in AS. Additionally, the Prostate Cancer Research International Active Surveillance (PRIAS) study demonstrated the reluctance of patients to undergo yearly biopsies, which may be also interpreted as patient non-compliance.[13] As might be expected, newly diagnosed low-risk PCa patients may probably have concerns about their treatment options and this situation may influence patient compliance. If AS is to be selected, it's clear that patients should be informed about the protocol in detail. In a longitudinal cohort study, it was reported that men preferring AS had greater knowledge and awareness of having lowrisk PCa, but also were less certain about their treatment preference, had a greater anxiety and preferred a shared treatment decision. [14] Compatibly, good communication and trustful relationship between patients and physicians were indicated as major factors for lowrisk PCa patients to enroll to AS protocol.[15,16]

In case scenarios; when asked for a primary treatment recommendation for very low/low risk PCa patients with life expectancy of $\geq 10$ years, only $54.1 \%$ of the physicians recommended AS. This result was nearly $20 \%$ and 50\% in Kim et al.s [10] and El Sebaaly et al.s [11] studies, respectively. Biased treatment suggestions of physicians in favor of their own expertises and influences of physicians' concerns on treatment decisions may explain these low rates.

When asked for a primary treatment recommendation for very low/low risk PCa patients with life expectancy of $<10$ years, $52.5 \%$ of the physicians rec- 
ommended AS. This result indirectly revealed that observation is not a generally accepted management strategy in this group of patients for PCa physicians who participated in this study. It may also be interpreted as awareness should be raised among physicians to distinguish AS from observation. Regardless of life expectancy of the patients, physicians didn't recommend AS for patients with favorable intermediate risk.

This study demonstrated that there was no majority consensus for a certain AS protocol among physicians. Although a great majority of the physicians preferred to use PSA test for AS protocol, there were differences about the optimal time interval for an evaluation with PSA test. To make a decision for AS, MPMRI were recommended by $75 \%$ of the participants. During follow-ups for AS, physicians' suggested rates of use of DRE, prostate biopsy and MPMRI were less than $70 \%$. In addition, physicians couldn't reach a consensus on the optimal time interval for an evaluation with DRE, prostate biopsy and MPMRI. These results were compatible with the findings of Ganz et al.[17] in which no consensus was reported on patient selection and follow-up protocols for AS. Significant heterogeneities in AS protocols were also reported in several different studies.[18-20] Therefore, it is important to establish a standart AS protocol to make the outcomes of the obtained data more valid and accurate.

\section{Limitations and Strength of the Study}

The limitations of our study are as follows. 1) Our questionnaire is not validated as there were no validated questionnaires on this topic. 2) Because this is a questionnaire study, we were only able to analyze limited data. There may be more questions to be addressed. 3) This study evaluated only perspectives of ROs, MOs and UROs towards AS and also did not assess the perspectives of the patients. 4) The number of medical oncologists was relatively lower than other physicians. The strength of our study is being the largest study evaluating the attitudes of ROs, MOs and UROs towards AS in the management of PCa. Above-mentioned (or even may be more) limitations were also present in other similar studies.

\section{Conclusion}

Being UROs or ROs rather than MOs and working in more academic or multidisciplinary conditions may lead PCa physicians to offer AS more. Biased treatment suggestions of physicians in favor of their own expertise and influences of physicians' concerns on treatment decisions may decrease the AS suggestion rates. It's critical to establish a certain AS protocol which could make obtained data outcomes more valid and accurate. It is also reasonable to raise awareness among physicians to distinguish AS from observation. In general, physicians don't recommend AS for PCa patients with favorable intermediate risk.

Acknowledgement: We would like to thank all the prostate cancer specialists who took the time to voluntarily complete our questionnaire.

Peer-review: Externally peer-reviewed.

Conflict of Interest: All authors declare that they have no conflict of interest.

Ethics Committee Approval: This study was approved by the Muğla Sttkı Koçman University Medical Faculty Ethics Committee (no. 147, date: 27.08.2019).

Financial Support: None declared.

Authorship contributions: Concept - G.T., A.A., İ.A., Ö.T., H.Ş.; Design - G.T., A.A., İ.A.; Supervision - G.T., A.A., İ.A., Ö.T., H.Ş.; Funding - G.T., A.A., İ.A., Ö.T., H.Ş.; Materials G.T., A.A., İ.A., Ö.T., H.Ş.; Data collection and/or processing - G.T., A.A., İ.A., Ö.T., H.Ş.; Data analysis and/or interpretation - G.T., A.A.; Literature search - G.T., A.A.; Writing G.T., A.A.; Critical review - G.T., A.A., İ.A., Ö.T., H.Ş.

\section{References}

1. Ferlay J, Colombet M, Soerjomataram I, Dyba T, Randi G, Bettio M, et al. Cancer incidence and mortality patterns in Europe: Estimates for 40 countries and 25 major cancers in 2018. Eur J Cancer 2018;103:356-87.

2. Garisto JD, Klotz L. Active Surveillance for Prostate Cancer: How to Do It Right. Oncology (Williston Park) 2017;31(5):333-40, 45 .

3. Butler SS, Loeb S, Cole AP, Zaslowe-Dude C, Muralidhar V, Kim DW, et al. United States trends in active surveillance or watchful waiting across patient socioeconomic status from 2010 to 2015. Prostate Cancer Prostatic Dis 2020;23(1):179-83.

4. Klotz L, Vesprini D, Sethukavalan P, Jethava V, Zhang L, Jain S, et al. Long-term follow-up of a large active surveillance cohort of patients with prostate cancer. J Clin Oncol 2015;33(3):272-7.

5. Meunier ME, Eyraud R, Sénéchal C, Gourtaud G, Roux V, Lanchon C, et al. Active Surveillance for Favorable Risk Prostate Cancer in African Caribbean Men: Results of a Prospective Study. J Urol 2017;197(5):1229-36.

6. Wilt TJ, Jones KM, Barry MJ, Andriole GL, Culkin D, Wheeler T, et al. Follow-up of Prostatectomy versus Observation for Early Prostate Cancer. N Engl J Med 
2017;377(2):132-42.

7. Hamdy FC, Donovan JL, Lane JA, Mason M, Metcalfe C, Holding P, et al; ProtecT Study Group. 10Year Outcomes after Monitoring, Surgery, or Radiotherapy for Localized Prostate Cancer. N Engl J Med 2016;375(15):1415-24.

8. Bill-Axelson A, Holmberg L, Garmo H, Rider JR, Taari $\mathrm{K}$, Busch $\mathrm{C}$, et al. Radical prostatectomy or watchful waiting in early prostate cancer. $\mathrm{N}$ Engl J Med 2014;370(10):932-42.

9. Thomsen FB, Røder MA, Hvarness H, Iversen P, Brasso K. Active surveillance can reduce overtreatment in patients with low-risk prostate cancer. Dan Med J 2013;60(2):A4575.

10. Kim SP, Gross CP, Shah ND, Tilburt JC, Konety B, Williams SB, et al. Perceptions of Barriers Towards Active Surveillance for Low-Risk Prostate Cancer: Results From a National Survey of Radiation Oncologists and Urologists. Ann Surg Oncol 2019;26(2):660-8.

11. El Sebaaly R, Mansour M, Labban M, Jaafar RF, Armache A, Mukherji D, et al. Survey on the practice of active surveillance for prostate cancer from the Middle East. Prostate Int 2020;8(1):41-8.

12. Davis K, Bellini P, Hagerman C, Zinar R, Leigh D, Hoffman R, et al. Physicians' Perceptions of Factors Influencing the Treatment Decision-making Process for Men With Low-risk Prostate Cancer. Urology 2017;107:86-95.

13. Bokhorst LP, Alberts AR, Rannikko A, Valdagni R, Pickles T, Kakehi Y, et al; PRIAS study group. Compliance Rates with the Prostate Cancer Research International Active Surveillance (PRIAS) Protocol and Disease Reclassification in Noncompliers. Eur Urol
2015;68(5):814-21.

14. Taylor KL, Hoffman RM, Davis KM, Luta G, Leimpeter A, Lobo T, et al. Treatment Preferences for Active Surveillance versus Active Treatment among Men with Low-Risk Prostate Cancer. Cancer Epidemiol Biomarkers Prev 2016;25(8):1240-50.

15. Gorin MA, Soloway CT, Eldefrawy A, Soloway MS. Factors that influence patient enrollment in active surveillance for low-risk prostate cancer. Urology 2011;77(3):588-91.

16. Kinsella N, Stattin P, Cahill D, Brown C, Bill-Axelson A, Bratt $\mathrm{O}$, et al. Factors Influencing Men's Choice of and Adherence to Active Surveillance for Low-risk Prostate Cancer: A Mixed-method Systematic Review. Eur Urol 2018;74(3):261-80.

17. Ganz PA, Barry JM, Burke W, Col NF, Corso PS, Dodson E, et al. National Institutes of Health State-of-theScience Conference: role of active surveillance in the management of men with localized prostate cancer. Ann Intern Med 2012;156(8):591-5.

18. Azmi A, Dillon RA, Borghesi S, Dunne M, Power RE, Marignol L, O'Neill BD. Active surveillance for low-risk prostate cancer: diversity of practice across Europe. Ir J Med Sci 2015;184(2):305-11.

19. Gorin MA, Eldefrawy A, Ekwenna O, Soloway MS. Active surveillance for low-risk prostate cancer: knowledge, acceptance and practice among urologists. Prostate Cancer Prostatic Dis 2012;15(2):177-81.

20. Loeb S, Carter HB, Schwartz M, Fagerlin A, Braithwaite RS, Lepor H. Heterogeneity in active surveillance protocols worldwide. Rev Urol 2014;16(4):202-3. 
Supplementary Table 1 Questionnaire for evaluating the attitudes of prostate cancer specialists towards active surveillance in the management of prostate cancer.

1. What is your medical specialty?

a. Radiation Oncologist

b. Medical Oncologist

c. Urologist

2. What type of hospital are you working in?

a. Public hospital (non-teaching)

b. University/Education and Research Hospital

c. Private Hospital/Clinic

3. What is your current educational status?

a. Resident/Fellow

b. Specialist

c. Academic member (Professor, Associated/Assistant Professor)

4. Please check the age group to which you belong:

a. $<30$ years

b. 30-50 years

c. $>50$ years

5. Please check your gender:

a. Female

b. Male

6. For how long have you been working in oncology field?

a. $0-5$ years

b. 6-10 years

c. $\geq 11$ years

7. Do you have a multidisciplinary uro-oncology board in your working area?

a. Yes

b. No

c. No, but I had before

8. In your opinion, which one is the biggest problem in the management of prostate cancer? (multiple choices can be selected)

a. Screening

b. Diagnosis

c. Treatment

d. Overtreatment

e. Other (please specify) ......

9. Do you suggest active surveillance to prostate cancer patients in your daily practice?

a. Yes

b. No

10.Do you think that you have enough knowledge about inclusion criterias of active surveillance for prostate cancer?

a. Yes

b. No

c. I'm not sure / No idea

11.What do you think about the application of active surveillance for prostate cancer patients? (multiple choices can be selected)

a. It may be beneficial for selected patients

b. I am disagree with the application of active surveillance for prostate cancer patients

c. I'm worried about following patients with active surveillance

d. I don't have enough knowledge and experience about active surveillance

e. Other (please specify) ...... 
12.Which criteria(s) do you take into consideration for active surveillance during your polyclinic evaluation? (multiple choices can be selected)
a. Life expectancy
b. Prostate cancer risk group
c. Sexual activity status of the patient
d. Patient's request and compliance
e. Other (please specify) .....

13. What is your main concern about active surveillance? (multiple choices can be selected)
a. No concern with it, I confidently use.
b. Local progression risk
c. Lymphatic/systemic metastasis risk
d. Patient's non-compliance (including irregular follow-up)
e. Lack of sufficient evidence to support active surveillance
f. Risk of losing a curative treatment opportunity
g. Other (please specify) .....

14.Which treatment would you recommend first for very low/low risk prostate cancer patients with life expectancy of $\geq 10$ years?
a. Radical Prostatectomy
b. Radiotherapy
c. Active surveillance
d. Observation
e. Other (please specify) ......

15. Which treatment would you recommend first for favorable intermediate risk prostate cancer patients with life expectancy of $\geq 10$ years?

a. Radical Prostatectomy

b. Radiotherapy

c. Active surveillance

d. Observation

e. Other (please specify) ......

16.Which treatment would you recommend first for very low/low risk prostate cancer patients with life expectancy of $<10$ years?

a. Radical Prostatectomy

b. Radiotherapy

c. Active surveillance

d. Observation

e. Other (please specify) .....

17. Which treatment would you recommend first for favorable intermediate risk prostate cancer patients with life expectancy of $<\mathbf{1 0}$ years?
a. Radical Prostatectomy
b. Radiotherapy
c. Active surveillance
d. Observation
e. Other (please specify) ......

18.For localized disease, which imaging modality do you use to make a decision for active surveillance?
a. Gallium-68 Prostate-Specific Membrane Antigen PET Imaging
b. Multiparametric Prostate Magnetic Resonance Imaging
c. Pelvic Computerized Tomography 
19.Which of the followings do you use for active surveillance protocol? (multiple choices can be selected)

a. Multiparametric Prostate Magnetic Resonance Imaging

b. Gallium-68 Prostate-Specific Membrane Antigen Pet Imaging

c. Digital Rectal Examination

d. Prostate Biopsy

e. Prostate-specific-antigen test

f. Total Body Bone Scintigraphy

g. Thorax Computerized Tomography

h. Other (please specify) ......

20.How often do you assess your active surveillance patient in the polyclinic, unless clinically indicated?

a. Every 6 weeks

b. Every 3 months

c. Every 6 months

d. Annually

e. Other (please specify) .....

21. How often do you evaluate your active surveillance patient with PSA test , unless clinically indicated?

a. Never

b. Every 3 months

c. Every 6 months

d. Annually

e. Every 2 years

f. Other (please specify) .....

22. How often do you evaluate your active surveillance patient with digital rectal examination , unless clinically indicated?

a. Never

b. Every 3 months

c. Every 6 months

d. Annually

e. Every 2 years

f. Other (please specify) .....

23. How often do you evaluate your active surveillance patient with prostate biopsy, unless clinically indicated?
a. Never
b. Every 3 months
c. Every 6 months
d. Annually
e. Every 2 years
f. Other (please specify) .....

24. How often do you evaluate your active surveillance patient with multiparametric prostate magnetic resonance imaging, unless clinically indicated?
a. Never
b. Every 3 months
c. Every 6 months
d. Annually
e. Every 2 years
f. Other (please specify) 